\title{
DETERMINACIÓN DE COMPUESTOS FENÓLICOS, FLAVONOIDES TOTALES Y CAPACIDAD ANTIOXIDANTE EN MIELES PERUANAS DE DIFERENTES FUENTES FLORALES
}

\author{
Ana María Muñoz Jáuregui ${ }^{1^{*}}$, Carlos Alvarado-Ortíz Ureta², Teresa Blanco Blasco², \\ Benjamín Castañeda Castañeda ${ }^{3}$, Julio Ruiz Quiroz ${ }^{4}$, Ángel Alvarado Yarasca ${ }^{3}$.
}

\section{RESUMEN}

Se realizó el estudio en doce mieles de diferentes marcas colectadas en supermercados de Lima, determinándose el contenido de compuestos fenólicos totales según Pérez et al, flavonoides totales por Zhishen et al, ensayo de 2-desoxi-D-ribosa por Sandoval et al, capacidad antioxidante por el método ABAP/ABTS, según Overveld et al., su efecto antioxidante sobre el anión superóxido según Bermúdez-Campos et al y contenido de fenólicos por HPLC según Muñoz et al.

La miel silvestre del callejón de Huaylas tuvo mayor contenido $(207,89 \mathrm{mg} / 100 \mathrm{~g})$ de fenólicos, mientras la multifloral de Piura, mayor contenido (3,839 mg QE/100g) de flavonoides totales y apigenina $(1,799 \mathrm{ppm})$, pero menor inhibición del anión superóxido $(53,21 \%)$, mientras la de eucalipto de la sierra central, mayor capacidad antioxidante por el método ABTS $(68,452 \mu \mathrm{g}$ TEAC / $100 \mathrm{~g})$ y de inhibición del anión superóxido (64,73\%). Por otro lado, la de Zapote en panal de Piura presentó menor contenido $(0,914 \mathrm{mg}$ QE/100g) de flavonoides totales, pero mayor inhibición $(54,80 \%)$ de radical oxhidrilos y de clorogénico $\left(0,866^{\mathrm{i}} \mathrm{mg} / \mathrm{kg}\right)$. Asimismo se encontró mayor contenido de ácido coumárico en la de algarrobo (1,572 ppm). Se concluyó que la miel contiene una gran capacidad antioxidante, relacionada al contenido de compuestos fenólicos, variando según su origen floral y procedencia.

Palabras clave: Fenólicos totales, flavonoides, miel, antioxidante

\section{DETERMINATION OF PHENOLIC COMPOUNDS, TOTAL FLAVONOIDS AND ANTIOXIDANT ACTIVITY IN PERUVIAN HONEY FROM DIFFERENT FLOWER'S SOURCES}

\begin{abstract}
Ana María Muñoz Jáuregui ${ }^{1 *}$, Carlos Alvarado-Ortiz Ureta², Teresa Blanco Blasco², Benjamín Castañeda Castañeda ${ }^{3}$, Julio Ruiz Quiroz ${ }^{4}$, Ángel Alvarado Yarasca ${ }^{3}$.
\end{abstract}

\section{ABSTRACT}

The study was realized in twelve different brands of honey collected from supermarkets in Lima, determining the content of total phenolic compounds according to Pérez et al, total

\footnotetext{
1 Escuela de Ciencia de los Alimentos. Facultad de Farmacia y Bioquímica UNMSM. Jr. Puno 1002. Lima, Perú.amariamj@yahoo.es

Escuela de Postgrado. Universidad San Ignacio de Loyola

Instituto de Investigación. Facultad de Medicina Humana, USMP

${ }^{4}$ Facultad de Farmacia y Bioquímica. UNMSM.
} 
flavonoids by Zhishen et al trial Desoxy-D-ribose by Sandoval et al, antioxidant capacity method ABAP / ABTS as Overveld et al., its antioxidant effect on superoxide as BermudezCampos et al and phenolic content by HPLC according to Muñoz et al.

Wild honey Huaylas had higher content $(207,89 \mathrm{mg} / 100 \mathrm{~g})$ of phenol, whereas the higher content from Piura multifloral (QE 3,839 mg / 100g) of total flavonoids and apigenin $(1,799$ $\mathrm{ppm}$ ) but less inhibition of superoxide anion $(53,21 \%)$, while the central mountain eucalyptus higher antioxidant capacity by ABTS method (TEAC 68,452 $\mathrm{mg} / 100 \mathrm{~g}$ ) and inhibition of superoxide anion (64,73\%). Furthermore the honeycomb Zapote Piura showed less content (QE $0,914 \mathrm{mg} / 100 \mathrm{~g})$ of total flavonoids but greater inhibition $(54,80 \%)$ of oxhidrilos radical and chlorogenic $(0,866 \mathrm{mg} / \mathrm{kg})$. Also higher content of coumaric found in carob $(1,572 \mathrm{ppm})$. It was concluded that honey contains a high antioxidant capacity related to the content of phenolic compounds varies by floral source and origin.

Key words: Total phenolics, flavonoids, honey, antioxidant

\section{INTRODUCCIÓN}

La miel es un producto de la colmena preparado por las abejas a partir de sustancias azucaradas, el néctar y otros derivados de muchas plantas ${ }^{1}$. La miel se compone principalmente de los azúcares glucosa y fructosa, y su tercer componente más grande es agua. También contiene muchos otros tipos de azúcares, así como ácidos, proteínas y minerales ${ }^{2}$.

Los fitoquímicos son sustancias que se encuentran en los alimentos de origen vegetal, biológicamente activos; se encuentran en las plantas y son ampliamente investigados por los científicos por su promoción de la salud. La miel tiene una amplia gama de fitoquímicos, como los polifenoles, que actúan como antioxidantes ${ }^{3}$.

Los antioxidantes son sustancias nutritivas y no nutritivas que pueden retardar o inhibir la oxidación y/o neutralizar los efectos perjudiciales de los radicales libres. En los seres humanos, el estrés oxidativo está implicado en un número cada vez mayor de enfermedades crónicas, como las cardiovasculares y el cáncer. Mayor contenido de antioxidantes en el organismo podría ayudar a proteger contra el daño celular y el desarrollo de enfermedades crónicas. La investigación indica que la miel contiene numeros antioxidantes fenólicos y nofenólicos; la cantidad y tipo dependen, en gran medida, de la fuente floral de la miel. Mieles más oscuras son generalmente más altas en contenido de antioxidantes que las mieles más claras; se ha demostrado ser similares en la capacidad antioxidante al de las frutas y vegetales ${ }^{4}$. Los polifenoles y ácidos fenólicos encontrados en la miel varían de acuerdo a las condiciones geográficas y climáticas. Algunos de ellos fueron reportados como un marcador específico del origen botánico de la miel. Se ha encontrado diferencias considerables, tanto en la composición como en el contenido de compuestos fenólicos de las diferentes mieles florales 5 . Los principales metabolitos en las mieles son: pinobanksina, crisina, hesperetina, luteolina, 3metil quercetina, isorramnetina, pinocembrina, dimetil cafeato, fenil etil cafeato, miricetina3,7,4',5'-metiléter, galangina, galangina-3-metiléter, tectocrisina, ácido elágico, 8metoxikaempferol, apigenina, dimetilalil cafeato, quercetina, kaempferol, pinobanksin-3acetato $^{6}$.

\section{PARTE EXPERIMENTAL}

\section{Muestra}

Las muestras fueron colectadas de los supermercados de la capital (Lima-Perú) y almacenadas a temperaturas de refrigeración en el Centro de Investigación de Bioquímica y Nutrición. 
Doce muestras de mieles fueron colectadas de diferente origen floral; entre ellas el algarrobo (Prosopis pallida), eucalipto (Eucalyptus globulus), faique (Acacia macracantha), zapote (Capparis angulata), vichayo (Capparis ovalifolia), pájaro bobo (Tesalia integrifolia), chilco (Bacharis Lanciolata), suelda y mano de ratón (tabla 1).

Tabla 1. Lista de muestras; su origen geográfico y botánico.

\begin{tabular}{lll}
\hline Mieles & Origen geográfico & Floración \\
\hline M001 & Callejón de Huaylas & Eucalipto \\
M002 & Callejón de Huaylas & Silvestre \\
M003 & Valles andinos & Algarrobo \\
M004 & Costa norte & Zapote silvestre \\
M005 & Sierra central & Eucalipto \\
M006 & Oxapampa & Silvestre \\
M007 & Oxapampa & $*$ \\
M008 & Costa norte, Piura & Multifloral Silvestre \\
M009 & Costa norte & Multifloral \\
M010 & Costa norte, Piura & Zapote \\
M017 & Costa norte & Eucalipto \\
M018 & Costa norte & Silvestre \\
\hline
\end{tabular}

*Floración no indica.

\section{Reactivos}

Ácido gálico, Folin-Ciocalteu, catequina, 1,1-diphenyl-2-picrylhydrazyl (DPPH), 2,2'Azobis (2-methylpropionamidine) dihydrochloride (ABAP), 2,2'-Azino-bis (3-ethylbenzothiazoline-6-sulfonic acid) (ABTS), 2-desoxi-D-ribosa, pirogalol; fueron obtenidos de Sigma (Sigma-Aldrich GmbH, Sternheim, Alemania).

\section{Métodos de Análisis}

\section{Polifenoles totales}

El contenido de polifenoles totales fue determinado mediante el método descrito por Pérez et $\mathrm{al}^{1}$. Se tomó de 0,5 a $0,6 \mathrm{~g}$ de miel aproximadamente; $25 \mu \mathrm{L}$ del extracto fue mezclado con 450 $\mu \mathrm{L}$ de agua desionizada y $1500 \mu \mathrm{L}$ de la solución $\mathrm{A}\left(1 \%\right.$ de SDS, $0,4 \% \mathrm{NaOH}, 2 \% \mathrm{de}_{2} \mathrm{CO}_{3}$, $0,16 \%$ tartrato de sodio y potasio); a la solución A, se adicionó $1000 \mu \mathrm{L}$ de $\mathrm{CuSO}_{4}$ al $4 \%$. La mezcla fue incubada a $37^{\circ} \mathrm{C}$ por 10 minutos. Luego se agregó $150 \mu \mathrm{L}$ del reactivo FolinCiocalteu $0,2 \mathrm{~N}$. La absorbancia fue medida a $750 \mathrm{~nm}$ y se utilizó ácido gálico como estándar de referencia.

\section{Flavonoides totales}

Los flavonoides totales fueron determinados por el método desarrollado por Zhishen et al ${ }^{7}$; una alícuota de $250 \mu \mathrm{L}$ del extracto de la muestra fue mezclada con $1000 \mu \mathrm{L}$ de agua desionizada; inmediatamente después se añadió $75 \mu \mathrm{L}$ de $\mathrm{NaNO}_{2}$ y se dejó reaccionar 5 minutos. Posteriormente, $75 \mu \mathrm{L}$ de $\mathrm{AlCl}_{3}$ al $10 \%$ fue adicionado y $500 \mu \mathrm{L}$ de $\mathrm{NaOH} 1 \mathrm{M}$. La mezcla fue centrifugada a 3500 r.p.m. durante 5 minutos. Los flavonoides totales fueron expresados en $\mathrm{mg}$ CAT/100 $\mathrm{g}$ de muestra. Las absorbancias fueron medidas a $510 \mathrm{~nm}$. 


\section{Actividad antioxidante}

\section{Ensayo de DPPH}

La actividad antioxidante se determinó mediante el método modificado por Brand-Williams

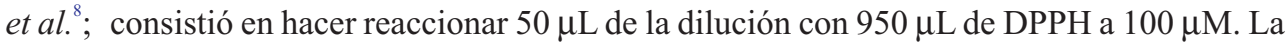
actividad antioxidante de las mieles fue expresada como $\mu \mathrm{g}$ de vitamina equivalente (VCEAC) por $100 \mathrm{~g}$ de muestra. El tiempo de reacción fue de 5 minutos y los valores de absorbancia fueron registrados a $515 \mathrm{~nm}$.

\section{Ensayo de ABTS/ABAP}

Las mediciones del potencial antioxidante por radical-trapping (TRAP) fue desarrollado por el ensayo ABAP/ABTS, usando el método descrito por Overveld et al. ${ }^{9}$. Los radicales ABTS fueron generados por descomposición termal del compuesto soluble en agua, 2,2'- azobis (2amidopropano) hidrocloride (ABAP), generando un compuesto de color verde debido a la formación estable del radical catiónico 2,2'-azinobis (3-etilbenzotiazolino-6-ácido sulfónico) $\mathrm{ABTS}^{+}$. El producto de oxidación es medido a $414 \mathrm{~nm}$. Los resultados fueron expresados como $\mu \mathrm{g}$ de trolox equivalente (TEAC) por $100 \mathrm{~g}$ de muestra.

\section{Ensayo de 2-desoxi-D-ribosa}

Para la determinación de captura del radical $\mathrm{OH}$ se usó el método utilizado por Sandoval et $a l^{10}$. Una cantidad de $200 \mu \mathrm{L}$ de 2-desoxy-D-ribosa $(2 \mathrm{mM}), 200 \mu \mathrm{L}$ de $\mathrm{KH}_{2} \mathrm{PO}_{4}(20 \mathrm{mM})$, $100 \mu \mathrm{L}$ de $\mathrm{FeCl}_{3}(100 \mu \mathrm{M}), 100 \mu \mathrm{l}$ de EDTA $(100 \mu \mathrm{M}), 100 \mu \mathrm{l}$ de $\mathrm{H}_{2} \mathrm{O}_{2}(1 \mathrm{mM}), 100 \mu \mathrm{L}$ de ácido ascórbico $(100 \mu \mathrm{M})$ y $50 \mu \mathrm{L}$ de muestra o estándar fueron mezclados. La reacción fue incubada por 2 horas a $37^{\circ} \mathrm{C}$. Después de este tiempo se adicionó TBA al $1 \%$ y se llevó a $100^{\circ} \mathrm{C}$ por 20 minutos. El producto color rosado generado por la reacción con TBA fue medido a $540 \mathrm{~nm}$.

\section{Ensayo del anión superóxido}

Se acondicionó los métodos descritos por Bermúdez-Camps et al. ${ }^{11}$. El medio de reacción contenía un volumen final de $3 \mathrm{~mL}$ de las siguientes concentraciones finales: $1000 \mu \mathrm{L}$ Tris- $\mathrm{HCl}$ de $50 \mathrm{mM}$ a pH 8,2; $1000 \mu \mathrm{L}$ EDTA a $1 \mathrm{mM}, 500 \mu \mathrm{L}$ de muestra y $500 \mu \mathrm{L}$ de pirogalol a $1 \mathrm{mM}$. Los valores de absorbancia se registraron a $420 \mathrm{~nm}$.

\section{Determinación de compuestos fenólicos por HPLC}

Se pesó $50 \mathrm{~g}$ de miel, y se diluyó esa muestra hasta completar $250 \mathrm{~mL}$ con agua acidificada con $\mathrm{HCl}$ a pH 2. Las columnas cromatográficas se llenaron con amberlita, y se llevó a empaquetar con agua destilada; luego se lavó la columna con un flujo de $200 \mathrm{~mL}$ de agua destilada seguido por $100 \mathrm{~mL}$ de agua acidificada con $\mathrm{HCl}$ a pH 2, luego de lo cual se añadió $250 \mathrm{~mL}$ de muestra previamente filtrada, después de lo cual se agregó $150 \mathrm{~mL}$ de agua acidificada con $\mathrm{HCl}$ a pH 2 y luego se agregó $300 \mathrm{~mL}$ de agua destilada para retirar los azúcares de la muestra presentes en la columna con la amberlita empaquetada y obteniendo un $\mathrm{pH}$ neutro; finalmente, se añadió 300 $\mathrm{mL}$ de metanol p.a. para la extracción de compuestos fenólicos presentes en la muestra (miel o polen). Para finalizar el proceso y trabajar las siguientes muestras se lavó la columna con 100 $\mathrm{mL}$ de agua destilada seguido por $150 \mathrm{~mL}$ de agua acidificada con $\mathrm{HCl}$ a pH 2, y se continuó con el mismo proceso indicado anteriormente. Una vez extraído los compuestos fenólicos en los balones de vidrio de base plana, se llevó a evaporar en baño maría a $80^{\circ} \mathrm{C}$ hasta sequedad; luego ese residuo se disolvió con $5 \mathrm{~mL}$ de agua destilada y se extrajo los compuestos fenólicos con éter dietílico realizando el lavado tres veces en una pera de decantación; ese último extracto se evaporó a temperatura ambiente en una campana de extracción y el residuo obtenido se reconstituyó con $2 \mathrm{~mL}$ de metanol grado $\mathrm{CG}$, para posteriormente ser analizadas en el HPLC. 


\section{RESULTADOS Y DISCUSIÓN}

Los resultados obtenidos en todas las tablas fueron evaluados estadísticamente mediante análisis de varianza (ANOVA) y al ser significativo su análisis, se realizó el test de comparación múltiple, aplicando la prueba de Duncan $(\mathrm{p}<0,05)$, donde las letras iguales indican que son muestras estadísticamente similares, mientras que letras diferentes indican que son muestras estadísticamente diferentes.

\section{Contenido de polifenoles totales y flavonoides totales}

En la tabla 2 se muestra el contenido de polifenoles totales en distintas muestras de miel expresado como ácido gálico (GAE) / $100 \mathrm{~g}$ muestra. La muestra 2 "miel silvestre" fue la que tuvo el mayor contenido de polifenoles totales, y la muestra 5 "miel eucalipto" la que tuvo menor contenido de polifenoles totales. El contenido hallado es superior a los encontrados por Muñoz y colaboradores ${ }^{6}$ en mieles chilena, cuyo valor máximo fue de $8,8 \mathrm{mg} / 100 \mathrm{~g}$. Por otro lado, Vit y colaboradores determinaron polifenoles en mieles producidos por la abeja Tetragonisca fiebrigi de Argentina y Paraguay, cuyos contenidos de compuestos fenólicos fue un promedio de $240,7 \mathrm{mg} / 100 \mathrm{~g}$ y $148,3 \mathrm{mg} / 100 \mathrm{~g}$ respectivamente. ${ }^{12}$

Tabla 2. Contenido de polifenoles totales en distintas muestras de miel expresado como ácido gálico (GAE) / $100 \mathrm{~g}$ muestra.

\begin{tabular}{clrcc}
\hline \hline \multirow{2}{*}{$\mathbf{N}^{\mathbf{2}}$} & Característica: Floración & \multicolumn{3}{c}{ mg GAE/100g muestra } \\
\cline { 3 - 5 } & & PROM & SD & Duncan \\
\hline 1 & Miel eucalipto & 180,37 & 2,56 & $\mathrm{~h}$ \\
2 & Miel silvestre & 207,89 & 2,18 & $\mathrm{i}$ \\
3 & Miel algarrobo & 121,81 & 1,85 & $\mathrm{~d}$ \\
4 & Miel zapote y otros & 143,72 & 2,73 & $\mathrm{f}$ \\
5 & Miel eucalipto & 83,15 & 4,09 & $\mathrm{a}$ \\
6 & Miel flora silvestre & 112,14 & 2,12 & $\mathrm{c}$ \\
7 & no indica & 102,72 & 0,91 & $\mathrm{~b}$ \\
8 & Miel multifloral de Piura & 145,28 & 1,89 & $\mathrm{f}$ \\
9 & Multifloral (3ra semana 16;8\%) & 177,05 & 2,69 & $\mathrm{~h}$ \\
10 & Zapote miel en panal de Piura & 134,87 & 1,20 & $\mathrm{e}$ \\
17 & Miel eucalipto & 150,08 & 3,32 & $\mathrm{~g}$ \\
18 & Miel silvestre & 150,91 & 1,69 & $\mathrm{~g}$ \\
\hline
\end{tabular}


Tabla 3. Contenido de flavonoides totales en distintas muestras de miel expresado como quercetina $(\mathrm{QE}) / 100 \mathrm{~g}$ muestra.

\begin{tabular}{clrcc}
\hline \hline \multirow{2}{*}{$\mathbf{N}^{\mathbf{*}}$} & \multirow{2}{*}{ Característica: Floración } & \multicolumn{3}{c}{ mg QE/ 100g muestra } \\
\cline { 3 - 5 } & & PROM & SD & Duncan \\
\hline 1 & Miel eucalipto & 1,4058 & 0,02 & $\mathrm{~b}$ \\
2 & Miel silvestre & 1,4032 & 0,08 & $\mathrm{~b}$ \\
3 & Miel algarrobo & 1,0063 & 0,01 & $\mathrm{a}$ \\
4 & Miel zapote y otros & 0,9651 & 0,01 & $\mathrm{a}$ \\
5 & Miel eucalipto & 1,5736 & 0,05 & $\mathrm{bc}$ \\
6 & Miel flora silvestre & 1,7563 & 0,06 & $\mathrm{c}$ \\
7 & no indica & 2,0755 & 0,07 & $\mathrm{~d}$ \\
8 & Multifloral de Piura & 3,8391 & 0,15 & $\mathrm{e}$ \\
9 & Multifloral (3ra semana 16.8\%) & 0,9692 & 0,02 & $\mathrm{a}$ \\
10 & Zapote miel en panal de Piura & 0.9139 & 0,02 & $\mathrm{a}$ \\
17 & Miel eucalipto & 1.6264 & 0,31 & $\mathrm{c}$ \\
18 & Miel silvestre & 1.4329 & 0,06 & $\mathrm{~b}$ \\
\hline
\end{tabular}

En la tabla 3 se muestra el contenido de flavonoides totales en distintas muestras de miel expresado como quercetina (QE) / $100 \mathrm{~g}$ muestra. El máximo contenido en mieles multifloral en Piura (3,83 mg QE/100g) fueron cercanos a los encontrados por Ciappini y colaboradores que arrojó valores promedio de : 3,28 $\pm 1,13 \mathrm{mg} Q \mathrm{QE} / 100 \mathrm{~g}$ de miel de "trébol", 3,95 $\pm 1,29 \mathrm{mg}$ QE/100 g de miel de "eucalipto" y 3,63 $\pm 0,74 \mathrm{mg}$ QE/100g para mieles de "alfalfa ${ }^{13}$.

La muestra 8 "miel multifloral" fue la que tuvo el mayor contenido de flavonoides totales, y las muestras 3, 4, 9 y 10 (miel algarrobo, miel zapote y otros, multifloral-3ra semana 16,8\% y miel de zapote en panal respectivamente) las que tuvieron estadísticamente menor contenido de flavonoides totales.

\section{Ensayo de DPPH}

En la tabla 4 se muestra la capacidad antioxidante medido por el ensayo de DPPH en distintas muestras de miel expresado como $\mu \mathrm{g}$ de vitamina C equivalente (VCEAC) / $100 \mathrm{~g}$ muestra. La muestra 9 "multifloral (3ra semana 16,8\%)" fue la que tuvo la mayor capacidad antioxidante, y la muestra de Oxapamapa, la que tuvo menor capacidad antioxidante; ambas medidas por el ensayo DPPH expresado como $\mu \mathrm{g}$ de vitamina C equivalente (VCEAC) / $100 \mathrm{~g}$ muestra. Otros estudios encontrados por Ciappiani y col., determinaron valores de capacidad antioxidante por el ensayo de DDPH expresado en trolox $(x=42,55 \pm 33,19 \mathrm{mg}$ $\mathrm{TE} / 100 \mathrm{~g}$ ) en mieles ${ }^{13}$. 
Tabla 4. Capacidad antioxidante medido por el ensayo de DPPH en distintas muestras de miel expresado como $\mu \mathrm{g}$ de vitamina $\mathrm{C}$ equivalente (VCEAC) / $100 \mathrm{~g}$ muestra.

\begin{tabular}{clccc}
\hline \hline \multirow{2}{*}{$\mathbf{N}^{\mathbf{0}}$} & Característica: Floración & \multicolumn{3}{c}{$\boldsymbol{\mu g} / \mathbf{1 0 0 g}$ muestra * } \\
\cline { 3 - 5 } & & PROM & SD & Duncan \\
\hline 1 & Miel eucalipto & 6,740 & 0,013 & $\mathrm{def}$ \\
2 & Miel silvestre & 6,769 & 0,001 & ef \\
3 & Miel algarrobo & 6,738 & 0,037 & $\mathrm{~d}$ \\
4 & Miel zapote y otros & 6,788 & 0,054 & $\mathrm{f}$ \\
5 & Miel eucalipto & 6,721 & 0,009 & $\mathrm{~d}$ \\
6 & Miel flora silvestre & 6,669 & 0,005 & $\mathrm{c}$ \\
7 & no indica & 6,370 & 0,010 & $\mathrm{a}$ \\
8 & Miel multifloral de Piura & 6,579 & 0,012 & $\mathrm{~b}$ \\
9 & Multifloral (3ra semana 16.8\%) & 6,850 & 0,056 & $\mathrm{~g}$ \\
10 & Zapote miel en panal de Piura & 6,778 & 0,006 & ef \\
17 & Miel eucalipto & 6,715 & 0,017 & $\mathrm{~d}$ \\
18 & Miel silvestre & 6,773 & 0,005 & ef \\
\hline
\end{tabular}

Tabla 5. Capacidad antioxidante medido por el ensayo de ABTS en distintas muestras de miel expresado como $\mu \mathrm{g}$ Trolox equivalente (TEAC) / $100 \mathrm{~g}$ muestra.

\begin{tabular}{clccc}
\hline \hline \multirow{2}{*}{$\mathbf{N}^{\mathbf{0}}$} & Característica: Floración & \multicolumn{3}{c}{$\boldsymbol{\mu g} / \mathbf{1 0 0 g}$ muestra * } \\
\cline { 3 - 5 } & & PROM & SD & Duncan \\
\hline 1 & Miel eucalipto & 37,464 & 0,032 & $\mathrm{f}$ \\
2 & Miel silvestre & 36,480 & 0,001 & $\mathrm{e}$ \\
3 & Miel algarrobo & 58,863 & 0,009 & $\mathrm{j}$ \\
4 & Miel zapote y otros & 28,747 & 0,002 & $\mathrm{a}$ \\
5 & Miel eucalipto & 68,452 & 0,026 & $\mathrm{1}$ \\
6 & Miel flora silvestre & 56,869 & 0,004 & $\mathrm{i}$ \\
7 & no indica & 47,617 & 0,005 & $\mathrm{~h}$ \\
8 & Miel multifloral de Piura & 67,825 & 0,005 & $\mathrm{k}$ \\
9 & Multifloral (3ra semana 16.8\%) & 33,740 & 0,010 & $\mathrm{~b}$ \\
10 & Zapote miel en panal de Piura & 34,257 & 0,012 & $\mathrm{c}$ \\
17 & Miel eucalipto & 34,417 & 0,001 & $\mathrm{~d}$ \\
18 & Miel silvestre & 40,694 & 0,008 & $\mathrm{~g}$ \\
\hline
\end{tabular}

\section{Ensayo de ABTS/ABAP}

En la tabla 5 se muestra la capacidad antioxidante medido por el ensayo de ABTS en distintas muestras de miel expresado como $\mu \mathrm{g}$ Trolox equivalente (TEAC) / $100 \mathrm{~g}$ muestra.

La muestra 5 "miel de eucalipto" fue la que tuvo la mayor capacidad antioxidante, y la muestra 4 "miel zapote y otros" la que tuvo menor capacidad antioxidante; ambas medido por el ensayo ABTS en distintas muestras de miel expresado como $\mu \mathrm{g}$ Trolox equivalente (TEAC) / $100 \mathrm{~g}$ muestra. Otros estudios encontrado por Ciappiani ${ }^{13}$ y col; indican contenidos de 102,02 $\pm 44,69 \mu \mathrm{g} \mathrm{TE} / 100 \mathrm{~g})$. 


\section{Ensayo de 2-desoxi-D-ribosa}

En la tabla 6 se muestra el ensayo de desoxi-D-ribosa en distintas muestras de miel expresado como porcentaje de inhibición para cada muestra. Las muestras 7 y 10 fueron las que tuvieron estadísticamente el mayor porcentaje de inhibición (54,62 y 54,80\% respectivamente), y la muestra 2 "miel silvestre" la que tuvo el menor porcentaje de inhibición; ambas medido por el ensayo 2-desoxi-Dribosa en distintas muestras de miel expresado como porcentaje de inhibición para cada muestra. Otros estudios realizados por Rodríguez y colaboradores en la miel proveniente de Apis miellifera, obtuvieron un mayor porcentaje de inhibición $(62,73 \%)^{14}$.

Tabla 6. Ensayo de desoxi-D-ribosa en distintas muestras de miel expresado como porcentaje de inhibición del radical $\mathrm{OH}$ para cada muestra.

\begin{tabular}{clccc}
\hline \hline \multirow{2}{*}{$\mathbf{N}^{\mathbf{*}}$} & \multirow{2}{*}{ Característica: Floración } & \multicolumn{3}{c}{$\%$ inhibición } \\
\cline { 3 - 5 } & & PROM & SD & Duncan \\
\hline 1 & Miel eucalipto & 31,56 & 1,89 & $\mathrm{~d}$ \\
2 & Miel silvestre & 1,72 & $0, .42$ & $\mathrm{a}$ \\
3 & Miel algarrobo & 33,11 & 3,79 & $\mathrm{~d}$ \\
4 & Miel zapote y otros & 30,57 & 1,04 & $\mathrm{~d}$ \\
5 & Miel eucalipto & 11,78 & 0,78 & $\mathrm{~b}$ \\
6 & Miel flora silvestre & 15,74 & $2, .93$ & $\mathrm{c}$ \\
7 & no indica & 54,62 & $1, .59$ & $\mathrm{f}$ \\
8 & Miel multifloral de Piura & 10,65 & 2.23 & $\mathrm{~b}$ \\
9 & Multifloral (3ra semana 16.8\%) & 43,61 & 0,83 & $\mathrm{e}$ \\
10 & Zapote miel en panal de Piura & 54,80 & 1,64 & $\mathrm{f}$ \\
17 & Miel eucalipto & 42,63 & 0,98 & $\mathrm{e}$ \\
18 & Miel silvestre & 45,19 & 2,69 & $\mathrm{e}$ \\
P* & Catequina & 79,23 & 1,17 & \\
\hline
\end{tabular}

P* corresponde al patrón "Catequina"

\section{Ensayo de anión superóxido}

En la tabla 7 se muestra el ensayo de anión superóxido en distintas muestras de miel expresado como porcentaje de inhibición para cada muestra.

Las muestras 1, 2, 3, 5, 6, 10, 17 y 18 fueron las que tuvieron estadísticamente el mayor porcentaje de inhibición del anión superoxido, siendo la muestra 17 de eucalipto la de mayor inhibición $(64,73 \%$ ) y las muestras 3, 4, 8 y 9 las que tuvieron, estadísticamente, el menor porcentaje de inhibición. Otros estudios realizados por Rodríguez y colaboradores obtuvieron un valor promedio de $69,06 \%$ de inhibición en miel proveniente de Apis miellifera $^{14}$. 
Tabla 7. Ensayo de anión superóxido en distintas muestras de miel expresado como porcentaje de inhibición para cada muestra.

\begin{tabular}{clccc}
\hline \hline \multirow{2}{*}{$\mathbf{N}^{\mathbf{0}}$} & Característica: Floración & \multicolumn{3}{c}{ \% inhibición } \\
\cline { 3 - 4 } & & PROM & SD & Duncan \\
\hline 1 & Miel eucalipto & 62,72 & 2,90 & $\mathrm{~cd}$ \\
2 & Miel silvestre & 62,56 & $3, .12$ & $\mathrm{~cd}$ \\
3 & Miel algarrobode valles andinos & 58,68 & 2,83 & $\mathrm{abcd}$ \\
4 & Miel zapote y otros & 58,25 & 3,24 & $\mathrm{abc}$ \\
5 & Miel eucalipto & 61,34 & 3,76 & $\mathrm{bcd}$ \\
6 & Miel flora silvestre & 59,43 & 3,77 & $\mathrm{bcd}$ \\
7 & no indica & 62,33 & 2,85 & $\mathrm{~cd}$ \\
8 & Miel multifloral de Piura & 53,21 & 3,17 & $\mathrm{a}$ \\
9 & Multifloral (3ra semana 16.8\%) & 56,06 & 3,40 & $\mathrm{ab}$ \\
10 & Zapote miel en panal de Piura & $61, .30$ & 3,36 & $\mathrm{bcd}$ \\
17 & Miel eucalipto & 64,73 & 3,29 & $\mathrm{c}$ \\
18 & Miel silvestre & $63, .33$ & 3,16 & $\mathrm{~cd}$ \\
\hline
\end{tabular}

\section{Determinación de compuestos fenólicos por HPLC en mieles}

En la tabla 8 se muestra el contenido de compuestos fenólicos: ácido clorogénico, siríngico, rutina, coumárico y apigenina en distintas muestras de miel expresado como $\mathrm{mg} / \mathrm{kg}$ de muestra; letras distintas indican que son muestras estadísticamente diferentes, siendo para el contenido de ácido clorogénico $(0,866 \mathrm{mg} / \mathrm{kg})$ la muestra 10 quien tuvo estadísticamente el mayor contenido, y las muestras 1 y 2 las que tuvieron, estadísticamente, los menores contenidos; ambas para el contenido de ácido clorogénico $(0,007$ y $0,009 \mathrm{mg} / \mathrm{kg})$.

El contenido de ácido siríngico en la muestra 3 "miel algarrobo" $(0,722 \mathrm{mg} / \mathrm{kg})$ fue la que obtuvo estadísticamente el mayor contenido, y la muestra 2 "miel silvestre" la de menor contenido $(0,018 \mathrm{mg} / \mathrm{kg})$.

El contenido de rutina en la muestra 18 "miel silvestre" $(0,121 \mathrm{mg} / \mathrm{kg})$ fue la que obtuvo estadísticamente el mayor contenido, y la muestra 5 "miel eucalipto" $(0,006 \mathrm{mg} / \mathrm{kg})$

la de menor contenido.

El contenido de coumárico en la muestra 3 "miel algarrobo" $(0,722 \mathrm{mg} / \mathrm{kg})$ fue la que obtuvo estadísticamente el mayor contenido, y la muestra $2(0,010 \mathrm{mg} / \mathrm{kg})$ la de menos contenido. El contenido de apigenina en la muestra 8 "miel multifloral" $(1,799 \mathrm{mg} / \mathrm{kg})$ fue la que obtuvo estadísticamente el mayor contenido, y la muestra de Oxapampa $(0,027 \mathrm{mg} / \mathrm{kg})$ la de menor contenido. Los flavonoides identificados en la miel son normalmente de los grupos de Flavanonas, flavononas y flavonoles, siendo importante la fuente de origen del néctar de la flor. 
Tabla 8. Contenido de compuestos fenólicos: ácido clorogénico, siríngico, rutina, coumárico y apigenina en distintas muestras de miel expresado como $\mathrm{mg} / \mathrm{kg}$ de muestra.

\begin{tabular}{llccccc}
\hline \hline $\mathbf{N}^{\circ}$ & Característica: Floración & \multicolumn{5}{c}{ Compuestos fenólicos } \\
\cline { 2 - 6 } & & Clorogénico & Siríngico & Rutina & Coumárico & Apigenina \\
\hline 1 & Miel eucalipto & $0,007^{\mathrm{a}}$ & $0,037^{\mathrm{b}}$ & $0,012^{\mathrm{c}}$ & $0,011^{\mathrm{a}}$ & $0,144^{\mathrm{c}}$ \\
2 & Miel silvestre & $0,009^{\mathrm{a}}$ & $0,018^{\mathrm{a}}$ & $0,034^{\mathrm{e}}$ & $0,010^{\mathrm{a}}$ & $0,346^{\mathrm{f}}$ \\
3 & Miel algarrobo de valles andinos & $0,029^{\mathrm{b}}$ & $0,722^{\mathrm{l}}$ & $0,073^{\mathrm{h}}$ & $1,572^{\mathrm{k}}$ & $0,191^{\mathrm{d}}$ \\
4 & Miel zapote y otros & $0,075^{\mathrm{d}}$ & $0,374^{\mathrm{i}}$ & $0,057^{\mathrm{f}}$ & $0,118^{\mathrm{e}}$ & $0,045^{\mathrm{b}}$ \\
5 & Miel eucalipto & $0,116^{\mathrm{e}}$ & $0,301^{\mathrm{f}}$ & $0,006^{\mathrm{a}}$ & $0,797^{\mathrm{i}}$ & $0,341^{\mathrm{f}}$ \\
6 & Miel flora silvestre & $0,041^{\mathrm{c}}$ & $0,340^{\mathrm{g}}$ & $0,008^{\mathrm{b}}$ & $0,925^{\mathrm{j}}$ & $0,472^{\mathrm{g}}$ \\
7 & no indica & $\mathrm{ND}$ & $0,365^{\mathrm{h}}$ & $0,065^{\mathrm{g}}$ & $0,075^{\mathrm{c}}$ & $0,027^{\mathrm{a}}$ \\
8 & Miel multifloral de Piura & $0,195^{\mathrm{g}}$ & $0,117^{\mathrm{c}}$ & $\mathrm{ND}$ & $0,261^{\mathrm{g}}$ & $1,799^{\mathrm{j}}$ \\
9 & Multifloral (3ra semana 16.8\%) & $0,762^{\mathrm{h}}$ & $0,490^{\mathrm{j}}$ & $0,086^{\mathrm{i}}$ & $0,112^{\mathrm{d}}$ & $0,224^{\mathrm{e}}$ \\
10 & Zapote miel en panal de Piura & $0,866^{\mathrm{i}}$ & $0 ., 24^{\mathrm{k}}$ & $\mathrm{ND}$ & $0,052^{\mathrm{b}}$ & $0,189^{\mathrm{d}}$ \\
17 & Miel eucalipto & $0,144^{\mathrm{f}}$ & $0,224^{\mathrm{d}}$ & $0,024^{\mathrm{d}}$ & $0,121^{\mathrm{f}}$ & $0,682^{\mathrm{i}}$ \\
18 & Miel silvestre & $0,036^{\mathrm{bc}}$ & $0,289^{\mathrm{e}}$ & $0,121^{\mathrm{j}}$ & $0,404^{\mathrm{h}}$ & $0,652^{\mathrm{h}}$ \\
\hline
\end{tabular}

\section{CONCLUSIONES}

La miel silvestre del callejón de Huaylas tuvo mayor contenido $(207,89 \mathrm{mg} / 100 \mathrm{~g})$ de fenólicos, mientras la multifloral de Piura mayor contenido (3,839 mg QE/ 100g ) de flavonoides totales y apigenina (1,799 ppm) pero menor inhibición del anión superóxido $(53,21 \%)$.

La miel de eucalipto de la sierra central tuvo mayor capacidad antioxidante por el método ABTS $(68,452 \mu \mathrm{g}$ TEAC/100g) y de inhibición del anión superóxido $(64,73 \%)$.

La miel de zapote en panal de Piura presentó menor contenido $(0,914 \mathrm{mg}$ QE/100g) de flavonoides totales pero mayor inhibición $(54,80 \%)$ de radical oxhidrilos y mayor contenido de clorogénico $\left(0,866^{\mathrm{i}} \mathrm{mg} / \mathrm{kg}\right)$.

Asimismo, se encontró mayor contenido de coumárico en la de algarrobo (1,572 ppm). Se concluyó que la miel contiene una gran capacidad antioxidante relacionada al contenido de compuestos fenólicos, variando según su origen floral y procedencia.

\section{AGRADECIMIENTOS}

Loa autores agradecen a la facultad de Medicina Humana de la USMP y la Empresa Apiarios Llona y Bojórquez, por el soporte dado al desarrollo de la presente investigación.

\section{BIBLIOGRAFÍA}

1. Pérez E, Rodriguez-Malaver A, Vit P. Antioxidant capacity of Venezuelan honey in wistar rat homogenates. Journal of Medicinal Food 2006; 9:510 - 516.

2. National Honey Board (NHB). Carbohydrates and the sweetness of honey. http://www.honey.com/downloads/carb.pdf. Release 10, 2009.

3. Jaganathan S, Mandal M. Antiproliferative effects of honey and of its polyphenols: A review. Journal of Biomedicine and Biotechnology 2009; doi:10.1155/2009/830616

4. National Honey Board.. Honey and antioxidants. National Honey Board Web site. http://www.honey.com/consumers/honeyhealth/nutritionresearch/antioxidants.asp. Release 10, 2009. 
5. Amiot M, Aubert S, Gonnet M, Tacchini M. Les composés phénoliques des miels: étude préliminaire sur l'identification et la quantification par familles. Apidologie. 1989; 20 : $115-125$.

6. Muñoz O, Copaja S, Speisky H, Peña R, Montenegro G. Contenido de flavonoides y compuestos fenólicos de mieles chilenas e índice antioxidante. Química Nova. 2007; 30: $848-851$.

7. Zhishen J, Mengcheng T, Jianming W. The determination of flavonoid contents in mulberry and their scavenging effects on superoxide radicals. Food Chemistry. 1999; 64: $555-559$.

8. Brand-Williams W, Cuvelier M, Berset, C.. Use of a free Radical method to Evaluate Antioxidant Activity. Lebensm. Wiss. Tol. 1995; 28: 25-30.

9. Overveld FWPC, Haenen GRMM, Rhemrev J, Vermeiden JPW, Bast A.. Tyrosine as important contributor to the antioxidant capacity of seminal plasma. Chemico-Biological Interactions. 2000; 127(2): $151-161$.

10. Sandoval M, Okuhama NN, Angeles FM. Técnicas de investigación para determinar la actividad antioxidativa y antiinflamatoria de plantas medicinales de la amazonía. 1st Internacional Workshop del 13 al 15 de agosto. Iquitos, Perú. 27 p. 2001.

11. Bermúdez-Camps I, Reyes-Hernández I, León-Fernández OS. Evaluación de la actividad antioxidante del propóleos de la región de Manzanillo. Provincia Granma. Cuba. IMBIOMED: Bioquímica. 2000;25(3): 69-74.

12. Vit P, Gutierrez M, Rodriguez- Malaver J, Aguilera G, Fernández-Díaz C, Tricios A. Comparación de mieles producidas por la abeja yateí( Tetragonisca fiebrigi) en Argentina y Paraguay. Acta Bioquím Clín Latinoam. 2009; 43 (2): 219-26.

13. Ciappini M, Stoppani F, Martinet R, alvarez M. Actividad antioxidante y contenido de compuestos fenólicos y favonoides en mieles de tréboles, eucalipto y alfalfa. Rev. Cienc. Tecnol. 2013;15(19):45-51.

14. Rodríguez A, Pérez E, Vit, P. Capacidad antioxidante de mieles venezolanas de los géneros Apis, Melipona y Tetragonisca, evaluada por tres métodos. INHRR [online]. 2007[acceso 26de noviembre de 2014]; 38(2):[13-18]. Disponible en http://www.scielo.org.ve/scielo.php?pid=S0798-04772007000200002\&script= sci_arttex 